International Joumal of Computational Geometry \& Applications

Vol. 3, No. 4 (1993) 467-468

(c) World Scientific Publishing Company

\title{
AUTHOR INDEX \\ VOLUME 3 (1993)
}

Abello, J. \& Eg̃eciog̃lu, Ö., Visibility graphs of staircase polygons with uniform step length

Agarwal, P.K. \& Sharir, M., Circular visibility from a point in a simple polygon

Asano, T., Dynamic programming on intervals

Atallah, M.J., Callahan, P.B. \& Goodrich, M.T., P-Complete geometric problems

Callahan, P.B., see Atallah, M.J.,

Carlsson, S., Nilsson, B.J. \& Ntafos, S., Optimum guard covers and $\mathbf{m}$-watchmen routes for restricted polygons

Chan, I.W. \& Friesen, D.K., Parallel algorithms for some dominance problems based on the PRAM model

Chan, K.-F. \& Lam, T.W., An on-line algorithm for navigating in an unknown environment

Chang, R.C., see Lee, H.-S.,

D'Amore, F. \& Franciosa, P.G., Separating sets of hyperrectangles (picture of)

D'Amore, F. \& Franciosa, P.G., Separating sets of hyperrectangles

Devillers, O., Simultaneous containment of several polygons:

Analysis of the contact configurations

Eades, P., Lin, T. \& Lin, X.M., Two tree drawing conventions

Ẽ̃eciog̃lu, Ö., see Abello, J.

Franciosa, P.G., see D'Amore, F.,

Franciosa, P.G., see D'amore, F.,

Friesen, D.K., see Chan, I.W.,

Gonzalez, T.F., Razzazi, M. \& Zheng, S.-Q., An efficient divide-and-conquer approximation algorithm for partitioning into D-boxes

Goodrich, M.T., see Atallah, M.J.,

Gotsman, C. \& Werman, M., Recognition of affine transformed planar curves by extremal geometric properties

Guibas, L.J., Hershberger, J.E., Mitchell, J.S.B. \& Snoeyink, J.S., Approximating polygons and subdivisions with minimum-link paths

Hershberger, J.E., see Guibas, L.J.,

Hirata, T., see Tan, X.,

Hsu, W.-L., Guest editor's foreword

3 (1993) 27-37

3 (1993) 1-25

3 (1993) 323-330

3 (1993) 443-462

3 (1993) 443-462

3 (1993) 85-105

3 (1993) 367-382

3 (1993) 227-244

3 (1993) 305-322

3 (1993) 345

3 (1993) 155-165

3 (1993) 429-442

3 (1993) 133-153

3 (1993) 27-37

3 (1993) 155-165

3 (1993) 345

3 (1993) 367-382

3 (1993) 417-428

3 (1993) 443-462

3 (1993) 183-202

3 (1993) 383-415

3 (1993) 383-415

3 (1993) 351-365

3 (1993) 225-226 
Inagaki, Y., see Tan, X.,

3 (1993) 351-365

Janardan, R., On maintaining the width and diameter of a planar point-set online

Janardan, R. \& Lopez, M., Generalized intersection searching problems

Lam, T.W., see Chan, K.-F.,

Lee, D.T. \& Sarrafzadeh, M., Maximum independent set of a permutation graph in $K$ tracks

Lee, H.-S. \& Chang, R.C., Regular Enumeration of grid points in an convex polygon

Lenhof, H.-P. \& Smid, M., An optimal construction method for generalized convex layers

Lin, T., see Eades, P.,

Lin, X.M., see Eades, P.,

Lopez, M., see Janardan, R.,

Mansour, Y., Park, J.K., Schieber, B. \& Sen, S., Improved selection in totally monotone arrays

Mitchell, J.S.B., see Guibas, L.J.,

Nilsson, B.J., see Carlsson, S.,

Ntafos, S., see Carlsson, S.,

Nussbaum, D. \& Sack, J.-R., Disassembling two-dimensional composite parts via translations

O'Rourke, J., Computational geometry column 18

O'Rourke, J., Computational geometry column 19

O'Rourke, J., Computational geometry column 20

O'Rourke, J., Computational geometry column 21

Park, J.K., see Mansour, Y.,

Preparata, F.P. \& Vitter, J.S., A simplified technique for hidden-line elimination in terrains

Razzazi, M., see Gonzalez, T.F.,

Sack, J.-R., see Nussbaum, D.,

Sarrafzadeh, M., see Lee. D.T.,

Schieber, B., see Mansour, Y.,

Sen, S., see Mansour, Y.,

Sharir, M., see Agarwal, P.K.

Shin, C.T.Y., A predictive algorithm for intersecting parametric polynomial surfaces

Smid, M., see Lenhof, H.-P.,

Snoeyink, J.S., see Guibas, L.J.,

Tan, X., Hirata, T. \& Inagaki, Y., An incremental algorithm for constructing shortest watchman routes

Vitter, J.S., see Preparata, F.P.,

Wagner, D., Optimal routing through dense channels

Werman, M., see Gotsman, C.,

Zheng, S.-Q., see Gonzalez, T.F.,

3 (1993) 331-344

3 (1993) 39-69

3 (1993) 227-244

3 (1993) 291-304

3 (1993) 305-322

3 (1993) 245-267

3 (1993) 133-153

3 (1993) 133-153

3 (1993) 39-69

3 (1993) 115-132

3 (1993) 383-415

3 (1993) 85-105

3 (1993) 85-105

3 (1993) 71-84

3 (1993) $107-113$

3 (1993) 221-224

3 (1993) 347-349

3 (1993) 463-465

3 (1993) 115-132

3 (1993) 167-181

3 (1993) 417-428

3 (1993) 71-84

3 (1993) 291-304

3 (1993) 115-132

3 (1993) 115-132

3 (1993) 1-25

3 (1993) 203-219

3 (1993) 245-267

3 (1993) 383-415

3 (1993) 351-365

3 (1993) 167-181

3 (1993) 269-289

3 (1993) 183-202

3 (1993) 417-428 